\title{
Theory of Diluted Magnetic Semiconductor Ferromagnetism
}

\author{
Jürgen König ${ }^{1}$, Hsiu-Hau Lin ${ }^{1,2}$, and Allan H. MacDonald ${ }^{1}$ \\ ${ }^{1}$ Department of Physics, Indiana University, Bloomington, IN 47405 \\ ${ }^{2}$ Department of Physics, National Tsing-Hua University, Hsinchu 300, Taiwan
}

\begin{abstract}
We present a theory of carrier-induced ferromagnetism in diluted magnetic semiconductors $\left(\mathrm{III}_{1-x} \mathrm{Mn}_{x} \mathrm{~V}\right)$ which allows for arbitrary itinerant-carrier spin polarization and dynamic correlations. Both ingredients are essential in identifying the system's elementary excitations and describing their properties. We find a branch of collective modes, in addition to the spin waves and Stoner continuum which occur in metallic ferromagnets, and predict that the low-temperature spin stiffness is independent of the strength of the exchange coupling between magnetic ions and itinerant carriers. We discuss the temperature dependence of the magnetization and the heat capacity.
\end{abstract}

75.30.Ds,75.40.Gb,75.50.Dd

Introduction. The recent discovery of carrier-induced ferromagnetism [1,2] in diluted magnetic semiconductors (DMS) has generated intense interest, in part because it opens the prospect of developing devices which combine information processing and storage functionalities in one material 3 9. Critical temperatures $T_{c}$ exceeding 100K have been realized [3] by using low-temperature molecular beam epitaxy growth to introduce a high concentration $c$ of randomly distributed $\mathrm{Mn}^{2+}$ ions in GaAs systems with a high hole density $c^{*}$. The tendency toward ferromagnetism and trends in the observed $T_{c}$ 's have been explained using a picture [10 14 in which uniform itinerant-carrier spin polarization mediates a longrange ferromagnetic interaction between the $\mathrm{Mn}^{2+}$ ions with spin $S=5 / 2$.

We present here the first theory which accounts for dynamic correlations in the ordered state and is able to describe its fundamental properties. We use a pathintegral formulation and, in the RKKY spirit of the previous theory, integrate out the itinerant carriers, and expand the effective action for the impurity spins up to quadratic order. In addition to the usual Goldstone spin waves, we find itinerant-carrier dominated collective excitations analogous to the optical spin-waves in a ferrimagnet. We also find that the spin stiffness is inversely proportional to the itinerant-carrier mass and independent of the strength of the exchange coupling between magnetic ions and itinerant carriers, in contrast to the meanfield critical temperature which is proportional to the itinerant-carrier mass and to the square of the exchange coupling. We extend our calculations to the critical temperature using an approximate self-consistent spin-wave scheme and address the temperature-dependent magnetization and specific heat.

Hamiltonian and effective action. We study a model which provides an accurate 15] description of Mn based zincblende DMS's. Magnetic ions with $S=5 / 2$ at positions $\vec{R}_{I}$ are antiferromagnetically coupled to valenceband carriers described by envelope functions,

$$
H=H_{0}+J_{p d} \int d^{3} r \vec{S}(\vec{r}) \cdot \vec{s}(\vec{r}),
$$

where $\vec{S}(\vec{r})=\sum_{I} \vec{S}_{I} \delta\left(\vec{r}-\vec{R}_{I}\right)$ is the impurity-spin density. The itinerant-carrier spin density is expressed in terms of carrier field operators by $\vec{s}(\vec{r})=$ $\frac{1}{2} \sum_{\sigma \sigma^{\prime}} \Psi_{\sigma}^{\dagger}(\vec{r}) \vec{\tau}_{\sigma \sigma^{\prime}} \Psi_{\sigma^{\prime}}(\vec{r})$. ( $\vec{\tau}$ is the vector of Pauli spin matrices.) $\quad H_{0}$ includes the valence-band envelopefunction Hamiltonian [16 and, if an external magnetic field $\vec{B}$ is present, the Zeeman energy,

$$
\begin{aligned}
H_{0}= & \int d^{3} r\left\{\sum_{\sigma} \hat{\Psi}_{\sigma}^{\dagger}(\vec{r})\left(-\frac{\hbar^{2} \vec{\nabla}^{2}}{2 m^{*}}-\mu^{*}\right) \hat{\Psi}_{\sigma}(\vec{r})\right. \\
& \left.-g \mu_{B} \vec{B} \cdot \vec{S}(\vec{r})-g^{*} \mu_{B} \vec{B} \cdot \vec{s}(\vec{r})\right\} .
\end{aligned}
$$

The effective mass, chemical potential, and $g$-factor of the itinerant carriers are labeled by $m^{*}, \mu^{*}$, and $g^{*}$. The model we use here is, thus, related to colossol magnetoresistance (CMR) materials 17 and identical to those for dense Kondo systems, which simplify when the itinerantcarrier density $c^{*}$ is much smaller than the magnetic ion density $c$ 18]. The fact that $c^{*} / c \ll 1$ in ferromagneticsemiconductor materials is essential to their ferromagnetism. Similar models have been used for ferromagnetism induced by magnetic ions in nearly ferromagnetic metals such as palladium [19].

We represent the impurity spins in terms of HolsteinPrimakoff (HP) bosons [20]. By coarse graining, the spin density $\vec{S}(\vec{r})$ can be replaced by a smooth function,

$$
\begin{aligned}
& S^{+}(\vec{r})=\left(\sqrt{2 c S-b^{\dagger}(\vec{r}) b(\vec{r})}\right) b(\vec{r}) \\
& S^{-}(\vec{r})=b^{\dagger}(\vec{r}) \sqrt{2 c S-b^{\dagger}(\vec{r}) b(\vec{r})} \\
& S^{z}(\vec{r})=c S-b^{\dagger}(\vec{r}) b(\vec{r})
\end{aligned}
$$

with bosonic fields $b^{\dagger}(\vec{r}), b(\vec{r})$. The partition function as a coherent-state path-integral in imaginary times reads,

$$
Z=\int \mathcal{D}[\bar{z} z] \mathcal{D}[\bar{\Psi} \Psi] e^{-\int_{0}^{\beta} d \tau L(\bar{z} z, \bar{\Psi} \Psi)}
$$


with $L=\int d^{3} r\left[\bar{z} \partial_{\tau} z+\sum_{\sigma} \bar{\Psi}_{\sigma} \partial_{\tau} \Psi_{\sigma}\right]+H(\bar{z} z, \bar{\Psi} \Psi)$. The bosonic (impurity spins) and fermionic (itinerant carriers) degrees of freedom are labeled by the complex variables $\bar{z}, z$ and the Grassmann numbers $\bar{\Psi}, \Psi$, respectively.

Since the Hamiltonian is bilinear in fermionic fields, we can integrate out the itinerant carriers and arrive at an effective description in terms of the localized spin density only, $Z=\int \mathcal{D}[\bar{z} z] \exp \left(-S_{\text {eff }}[\bar{z} z]\right)$ with the action

$$
\begin{aligned}
S_{\mathrm{eff}}[\bar{z} z]=\int_{0}^{\beta} & d \tau \int d^{3} r\left[\bar{z} \partial_{\tau} z-g \mu_{B} B(c S-\bar{z} z)\right] \\
& -\ln \operatorname{det}\left[\left(G^{M F}\right)^{-1}+\delta G^{-1}(\bar{z} z)\right] .
\end{aligned}
$$

Here, we have already split the total kernel $G^{-1}$ into a mean-field part $\left(G^{M F}\right)^{-1}$ and a fluctuating part $\delta G^{-1}$,

$$
\begin{aligned}
\left(G^{M F}\right)^{-1} & =\left(\partial_{\tau}-\frac{\hbar^{2} \vec{\nabla}^{2}}{2 m^{*}}-\mu^{*}\right) \mathbf{1}+\frac{\Delta}{2} \tau^{z} \\
\delta G^{-1} & =\frac{J_{p d}}{2}\left[\left(z \tau^{-}+\bar{z} \tau^{+}\right) \sqrt{2 c S-\bar{z} z}-\bar{z} z \tau^{z}\right]
\end{aligned}
$$

where $\Delta=c J_{p d} S-g^{*} \mu_{B} B$ is the zero-temperature spinsplitting gap for the itinerant carriers. The physics of the itinerant carriers is embedded in the effective action of the magnetic ions. It is responsible for the retarded and non-local character of the interactions between magnetic ions, which is described here for the first time.

Independent spin-wave theory. The independent spinwave theory, which is a good approximation at low temperatures, is obtained by expanding Eq. (7) up to quadratic order in $z$. We find (in Fourier representation)

$$
S_{\mathrm{eff}}[\bar{z} z]=\frac{1}{\beta V} \sum_{|\vec{p}|<p_{c}, m} \bar{z}\left(\vec{p}, \nu_{m}\right) D^{-1}\left(\vec{p}, \nu_{m}\right) z\left(\vec{p}, \nu_{m}\right),
$$

in addition to the temperature-dependent mean-field contribution. A Debye cutoff $\left(p_{c}^{3}=6 \pi^{2} c\right)$ ensures that we include the correct number of magnetic ion degrees of freedom. The kernel of the quadratic action is the inverse of the spin-wave propagator,

$$
\begin{aligned}
& D^{-1}\left(\vec{p}, \nu_{m}\right)=-i \nu_{m}+g \mu_{B} B+J_{p d} n^{*} \\
& \quad+\frac{c J_{p d}^{2} S}{2 \beta V} \sum_{n, \vec{k}} G_{\uparrow}^{M F}\left(\vec{k}, \omega_{n}\right) G_{\downarrow}^{M F}\left(\vec{k}+\vec{p}, \omega_{n}+\nu_{m}\right)
\end{aligned}
$$

with the mean-field itinerant carrier Green's function $G_{\sigma}^{M F}\left(\vec{k}, \omega_{n}\right)=-\left[i \omega_{n}-\left(\epsilon_{\vec{k}}+\sigma \Delta / 2-\mu^{*}\right)\right]^{-1}$. The mean-field spin density is denoted by $n^{*}=\left(n_{\downarrow}-n_{\uparrow}\right) / 2$, and $\epsilon_{\vec{k}}=\hbar^{2} k^{2} /\left(2 m^{*}\right)$.

Excitations. We obtain the spectral density of the spinfluctuation propagator by analytical continuation, $i \nu_{m} \rightarrow$ $\Omega+i 0^{+}$and $A(\vec{p}, \Omega)=\operatorname{Im} D(\vec{p}, \Omega) / \pi$. We find three different types of spin excitations. In all figures we take $B=0$ and use as typical parameters [3] $m^{*}=0.5 m_{e}$, $J_{p d}=0.15 \mathrm{eVnm}^{3}$, and $c=1 \mathrm{~nm}^{-3}$, where $m_{e}$ is the free-electron mass. For these parameters the mean-field itinerant-carrier system is fully polarized at $T=0$.

i) Our model has a gapless Goldstone-mode branch (see Fig. 11) reflecting the spontaneous breaking of rotational symmetry [21]. Expansion of the $T=0$ propagator at $\Delta>\epsilon_{F}$, where $\epsilon_{F}$ is the Fermi energy of the majority-spin band, yields for small and large momenta the dispersion of the collective modes

$$
\Omega_{p}^{(1)}=\frac{x}{1-x} \epsilon_{p}\left(1-\frac{4 \epsilon_{F}}{5 \Delta}\right)+\mathcal{O}\left(p^{4}\right)
$$

and $\Omega_{p}^{(1)}=x \Delta\left(1-\Delta / \epsilon_{p}\right)+\mathcal{O}\left(1 / p^{4}\right)$. At short wavelengths we obtain the mean-field result $x \Delta$, the spinsplitting of a magnetic ion in the effective field produced by fully spin-polarized itinerant carriers. Note that the itinerant-carrier and magnetic-ion mean-field spin splittings differ by a factor of $x=c^{*} /(2 c S) \ll 1$. At long wavelengths the magnon dispersion in an isotropic ferromagnet is proportional the spin stiffness $\rho$ divided by the magnetization $M$. In the adiabatic limit [17,22], $\epsilon_{F} \ll \Delta$, our long-wavelength result reflects a spin-stiffness due entirely to the increase in kinetic energy of a fully spinpolarized band when the orientation has a spatial dependence, $\rho=c^{*} \hbar^{2} /\left(4 m^{*}\right)$, and a magnetization $M$ which has opposing contributions from magnetic ions and itinerant carriers, $M=c S-c^{*} / 2=c S(1-x)$. In this limit, the mean-field critical temperature and the spin stiffness have opposite dependences on the itinerant-carrier mass.

The low-energy mode describes spin waves in the localimpurity system. This contrasts with the case of ferromagnetism induced by local moments [19] where the low-energy mode is primarily in the free-carrier part.

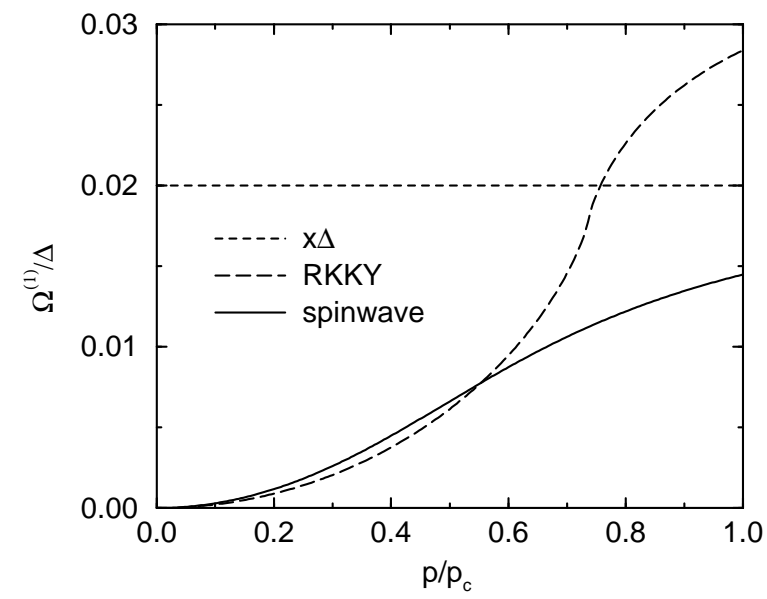

FIG. 1. Spin-wave dispersion for $c^{*}=0.1 \mathrm{~nm}^{-3}$. The short wavelength limit is the mean-field result $x \Delta$. For comparison, we show also the result obtained from an RKKY picture.

ii) We find a continuum of Stoner spin-flip particle-hole excitations. They correspond to flipping a single spin in the itinerant-carrier system and, therefore, occur at much 
larger energies $\sim \Delta$ (see Fig. 2). For $\Delta>\epsilon_{F}$ and zero temperature, all these excitations carry $\operatorname{spin} S^{z}=+1$, i.e., increase the spin polarization, and therefore turn up at negative frequencies in the boson propagator we study. (When $\Delta<\epsilon_{F}$, excitations with both $S^{z}=+1$ and $S^{z}=-1$ contribute to the spectral function.) This continuum lies between the curves $-\Delta-\epsilon_{p} \pm 2 \sqrt{\epsilon_{p} \epsilon_{F}}$ and for $\Delta<\epsilon_{F}$ also between $-\Delta+\epsilon_{p} \pm 2 \sqrt{\epsilon_{p}\left(\epsilon_{F}-\Delta\right)}$.

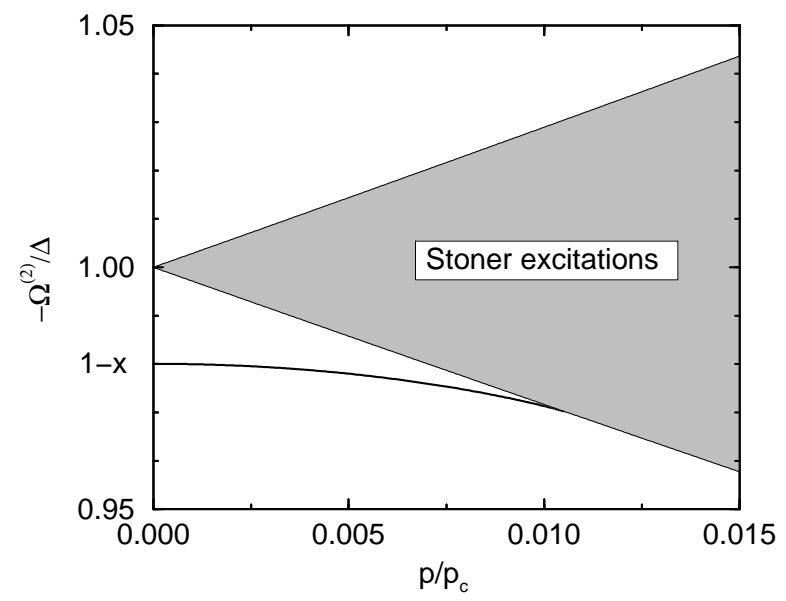

FIG. 2. Stoner excitations and collective modes in the free-carrier system for $c^{*}=0.1 \mathrm{~nm}^{-3}$.

iii) We find additional collective modes associated primarily with the itinerant-carrier system at energies below the Stoner continuum (see Fig. 2). At $T=0$ we obtain

$$
-\Omega_{p}^{(2)}=\Delta(1-x)-\frac{1}{1-x} \epsilon_{p}\left(\frac{4 \epsilon_{F}}{5 x \Delta}-1\right)+\mathcal{O}\left(p^{4}\right)
$$

for $\Delta>\epsilon_{F}$. The spectral weight of these modes is $-x /(1-x)$ at zero momentum.

The finite spectral weight at negative energies indicates that the ground state is not fully spin polarized because of quantum fluctuations.

Comparison to RKKY picture. For comparison we evaluate the $T=0$ magnon dispersion assuming an RKKY interaction between magnetic ions. This approximation results from our theory if we neglect the spin polarization in the itinerant carriers and evaluate the static limit of the resulting spin-wave propagator. The Stoner excitations and magnons shown in Fig. 2 do not emerge and the spin-wave dispersion (Fig. 11) is incorrect except for the limit $\Delta \ll \epsilon_{F}$. While the RKKY picture does, in some circumstances, provide a realistic estimate of $T_{c}$, it completely fails as a theory of the ferromagnetic state.

Comparison to a ferrimagnet. Some features of the excitation spectrum are, not coincidentally, like those of a localized spin ferrimagnet with antiferromagnetically coupled large spin $S$ (corresponding to the magnetic ions) and small spin $s$ (corresponding to the free carriers) subsystems on a bipartite cubic lattice. Representing the spins by HP bosons and expanding up to quadratic order, we either diagonalize the resulting Hamiltonian directly or, as in our ferromagnetic-semiconductor calculations, integrate out the smaller spins using a path-integral formulation to obtain equivalent results. We find that

$$
\Omega_{\vec{p}}^{(1) /(2)}=\frac{\Delta}{2}\left[-(1-x) \pm \sqrt{(1-x)^{2}+4 x \gamma_{\vec{p}}}\right]
$$

with $x=s / S$ and $\Delta=6 J S$, where $J$ is the exchange coupling, and $\gamma_{\vec{p}}=(1 / 3) \sum_{i}\left[1-\cos \left(p_{i} a\right)\right]$ with lattice constant $a$. We recover two collective modes, the coupled spin waves of the two subsystems. One is gapless, the other one gapped with $\Delta(1-x)$. The bandwidth is $x \Delta$, and the spectral weights at zero momentum are $1 /(1-x)$ and $-x /(1-x)$, respectively, as in our model.

Self-consistent scheme. Near the transition temperature $T_{c}$, the spin-wave density is of the order of $c S$, and expanding around a fully-polarized state is not a good starting point. Instead, we adopt a scheme which accounts self-consistently for the reduction of spin density in the localized impurity and itinerant carrier subsystems [23]. Our approach is motivated by the Weiss mean-field theory, which is expected to be accurate for a model with static long-range interactions between the magnetic ions. This model can be obtained in our approach by taking the Ising limit (i.e., replacing $\vec{S} \cdot \vec{s}$ by $S^{z} s^{z}$ ) and letting the mean-field itinerant-carrier spin splitting, $\Delta(T)=J_{p d}\left\langle S^{z}\right\rangle$, reflect the thermal suppression of the impurity-spin density $\left\langle S^{z}\right\rangle$. In this simple model the spin-wave spectrum is independent of momentum, $\Omega_{p}(T)=J_{p d} n^{*}(T)$, allowing a unitary transformation to spin-wave eigenstates with a site label. The constraint on the number of spin bosons $(\leq 2 S)$ on a site is then easily applied and we obtain

$$
\begin{aligned}
& \left\langle S^{z}\right\rangle=\frac{1}{V} \sum_{|\vec{p}|<p_{c}} S B_{S}\left(\beta S \Omega_{p}\right) \\
& =\frac{1}{V} \sum_{|\vec{p}|<p_{c}}\left\{S-n\left(\Omega_{p}\right)+(2 S+1) n\left[(2 S+1) \Omega_{p}\right]\right\},
\end{aligned}
$$

where $B_{S}(x)$ is the Brillouin function, and $n(\omega)$ is the Bose function. The first two terms in the second form of Eq. (15) give the spin density obtained by treating spin waves as bosons, while the last term is the correction from spin kinematics which rules out unphysical states.

Our self-consistent spin-wave approximation consists of using Eq. (15) when $\Omega_{p}$ is $p$-dependent (see Fig. 3). Although the characteristic $T^{3 / 2}$ law for the localizedions magnetization is recovered at low temperatures, the prefactor is reduced by $1-(2 S+1)^{-1 / 2}$, compared to the correct [24 value of linearized spin-wave theory. That is, constraining boson populations in momentum space is too restrictive at low temperatures, although the error is not serious for $S=5 / 2$. The transition temperature 
of the self-consistent spin-wave theory is lower than the estimate from Weiss mean-field theory.

The presence of spin waves also shows up in the specific heat $C_{V}$, which we calculate within the same scheme (see Fig. (4). Calculating the entropy $S$ we obtain

$$
C_{V}=\frac{T}{V} \frac{d S}{d T}=\frac{1}{V} \sum_{|\vec{p}|<p_{c}} \Omega_{p}(T)\left(\frac{d N_{p}}{d T}\right),
$$

where $N_{p}=S-S B_{S}\left(\beta S \Omega_{p}\right)$ is the average number of spin bosons at each momentum. The specific heat from the kinetic energy of itinerant carriers turns out to be negligibly small. The specific heat of magnetic ions $C_{V}$ is proportional to $T^{3 / 2}$ at low temperature, shows a Schottky-like anomaly, and has a jump at the transition temperature. We estimate that the jump is $5 \%$ of the lattice specific heat when $T_{c} \sim 100 \mathrm{~K}$, suggesting that this should be observable.

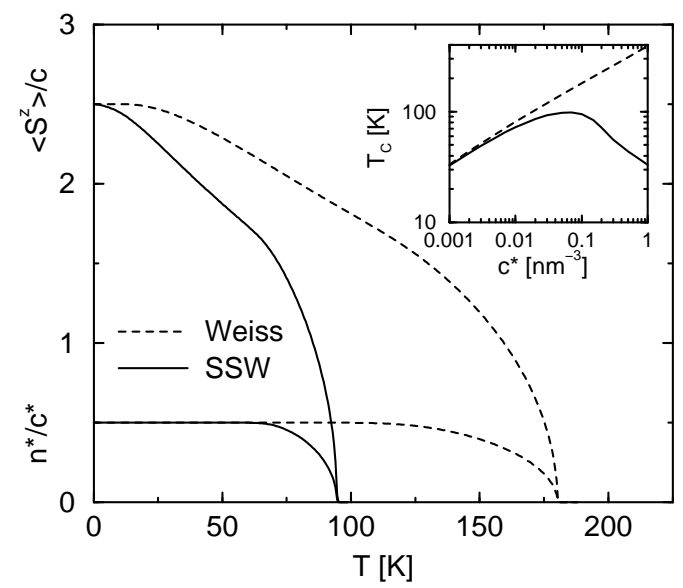

FIG. 3. Average impurity and itinerant-carrier spin as a function of temperature for $c^{*}=0.1 \mathrm{~nm}^{-3}$ in Weiss mean-field (Weiss) and self-consistent spin-wave theory (SSW). Inset: $T_{c}$ as a function of $c^{*}$.

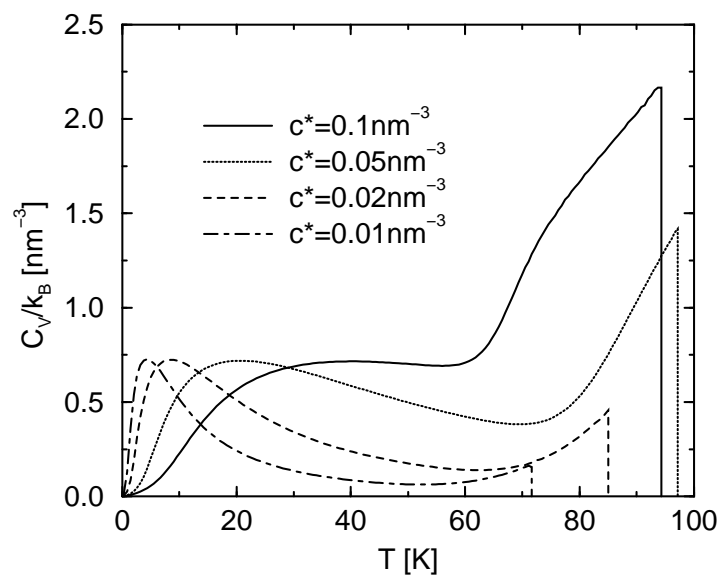

FIG. 4. Specific heat due to spin waves.
We acknowledge useful discussions with M. Abolfath, B. Beschoten, A. Burkov, J. Furdyna, S. Girvin, and T. Jungwirth. This work was supported by the Deutsche Forschungsgemeinschaft under grant KO 1987-1/1 and by the National Science Foundation, DMR-9714055.

[1] T. Story, R.R. Gałązka, R.B. Frankel, and P.A. Wolff, Phys. Rev. Lett. 56, 777 (1986).

[2] H. Ohno et al., Phys. Rev. Lett. 68, 2664 (1992); H. Ohno et al., Appl. Phys. Lett. 69, 363 (1996).

[3] H. Ohno, J. of Magnetism and Magnetic Materials 200, 110 (1999); Science 281, 951 (1998).

[4] G.A. Prinz, Science 282, 1660 (1998).

[5] T. Hayashi et al., J. Appl. Phys. 83, 6551 (1998).

[6] T.M. Pekarek, B.C. Crooker, I. Miotkowski, and A.K. Ramdas, J. Appl. Phys. 83, 6557 (1998).

[7] A. Van Esch et al., J. Phys. Condensed Matter 9, L361 (1997); A. Van Esch et al., Phys. Rev. B 56, 13103 (1997).

[8] A. Oiwa et al., Phys. Rev. B 59, 5826 (1999); F. Matsukura, H. Ohno, A. Shen, and Y. Sugawara, Phys. Rev. B 57, R2037 (1998).

[9] B. Beschoten et al., Phys. Rev. Lett. 83, 3073 (1999).

[10] T. Dietl, A. Haury, and Y.M. d'Aubigné, Phys. Rev. B 55, R3347 (1997).

[11] M. Takahashi, Phys. Rev. B 56, 7389 (1997).

[12] T. Jungwirth, W.A. Atkinson, B.H. Lee, and A.H. MacDonald, Phys. Rev. B 59, 9818 (1999).

[13] T. Dietl et al., Science 287, 1019 (2000).

[14] B.H. Lee, T. Jungwirth, and A.H. MacDonald, condmat/0001391, Phys. Rev. B 61, June 15 issue (2000).

[15] The central cell corrections required for an accurate envelope-function description of an isolated $\mathrm{Mn}^{2+}$ ion in GaAs [A.K. Bhattacharjee and C. Benoit à la Guillaume, Solid State Commun. 113, 17 (2000)] will be reduced by free-carrier screening.

[16] To simplify the present discussion we use a generic singleband model and neglect interactions between the free carriers as well as the antiferromagnetic Mn-Mn interaction. In quantitative calculations for particular materials it is necessary to account for enhanced ferromagnetism due to interactions [12], realistic heavy and light hole bands, and at high carrier densities, also the spin-orbit split-off band 13. These important effects will be discussed separately [J. König, H. Lin, and A.H. MacDonald (to be published)].

[17] A.J. Millis, P.B. Littlewood, and B.I. Shraiman, Phys. Rev. Lett. 74, 5144 (1995).

[18] M. Sigrist, H.Tsunetsugu, and K. Ueda, Phys. Rev. Lett. 67, 2211 (1991).

[19] S. Doniach and E.P. Wohlfarth, Proc. R. Soc. London Ser. A 296, 442 (1967).

[20] A. Auerbach, Interacting Electrons and Quantum Magnetism (Springer, New York, 1994).

[21] Because of spin-orbit coupling in the valence bands, the 
spin-wave spectrum in realistic models will have a strainand disorder-dependent gap [M. Abolfath, J. Brum, T. Jungwirth, and A.H. MacDonald (to be published)].

[22] E.L. Nagaev, Phys. Rev. B 58, 827 (1998).

[23] In the following, we neglect Stoner excitations and magnons in the itinerant carriers, i.e., we assume sharp spin-wave excitations with spectral weight one.

[24] F.J. Dyson, Phys. Rev. 102, 1230 (1956). 